\title{
Producción de Carbón Activado a partir de Precursores Carbonosos del Departamento del Cesar, Colombia
}

\author{
Marlon Bastidas*, Lina M. Buelvas, María I. Márquez y Kellys Rodríguez \\ Universidad Popular del Cesar, Centro de investigación y desarrollo tecnológico del carbón, Grupo \\ de Energías Alternativas y Biomasa, Bloque A, Oficina 205, Sede Hurtado, Salida a Patillal, \\ Valledupar, Cesar-Colombia (e-mail: mbastidasbarranco@gmail.com).
}

Recibido May. 25, 2009; Aceptado Jul. 13, 2009; Versión Final recibida Sep. 02, 2009

*autor a quien debe ser dirigida la correspondencia

\section{Resumen}

En este trabajo se realizó el montaje de una unidad experimental de activación para la obtención de carbones activados a partir de carbón bituminoso, endocarpio de coco y endocarpio de palma. El carbón bituminoso y los materiales biomásicos empleados provienen del Departamento del Cesar (Colombia) y fueron activados en un horno de atmósfera controlada. Los valores del área superficial específica de las muestras activadas fueron determinados a partir de las isotermas de $\mathrm{N}_{2}$. Los materiales carbonosos activados se emplearon en la adsorción de fenol en solución acuosa a diferentes concentraciones. El carbón activado obtenido del endocarpio de coco, alcanzó la mayor área superficial BET de $1200 \mathrm{~m}^{2} / \mathrm{g}$, y adsorbió la mayor cantidad de fenol: Sin embargo, la experimentación con otros materiales, demostró que grupos básicos contenidos en el material adsorbente y su microporosidad son factores determinantes para la adsorción de fenol.

Palabras clave: carbón activado, endocarpio de coco, endocarpio de palma, adsorción de fenol

\section{Activated Carbon Production from Carbonaceous Precursors of the Department of Cesar, Colombia}

\begin{abstract}
This work was conducted on an experimental assembly to obtain activated carbons from bituminous coal, and coconut and palm endocarp. Bituminous coal and biomass materials used come from the Department of Cesar (Colombia) and were activated in a controlled atmosphere furnace. The values of specific surface area of the activated samples were determined from the $\mathrm{N}_{2}$ adsorption isotherms. Activated coal from carbonaceous materials used in the adsorption of phenol in aqueous solution at different concentrations. Activated carbon obtained from coconut endocarp, reached higher BET surface area of $1200 \mathrm{~m}^{2} / \mathrm{g}$, and the greatest amount of adsorbed phenol. However, experiments with other materials showed that the basic groups contained in the adsorbent material and its microporosity are the determining factors for phenol adsorption.
\end{abstract}

Keywords: activated carbon, coconut endocarp, coconut endocarp, phenol adsorption 


\section{INTRODUCCIÓN}

El carbón activado es un adsorbente preparado a partir de materiales carbonosos que se caracteriza por poseer una alta superficie interna, variedad de grupos funcionales y una buena distribución de poros, propiedades que le permiten atrapar una gran diversidad de moléculas. La preparación de este tipo de material se lleva a cabo a través de procesos físicos o químicos, mediante la interacción con gases o la adición de químicos, respectivamente. Los carbones activados son utilizados generalmente en procesos de descontaminación de aguas, recuperación de solventes, control de emisiones, decoloración de líquidos, eliminación de olores, soportes catalíticos, entre otros procesos (Rodríguez, 2005; Bandosz, 2006). Entre los contaminantes de aguas más comunes se encuentran los compuestos fenólicos, presentes ampliamente en los efluentes de industrias refinadoras de petróleo, carboníferas, transformadoras de plástico, cuero, pintura, farmacéuticas, y del acero. La adsorción de compuestos fenólicos con carbón activado es uno de los métodos más usados, especialmente para efluentes con concentraciones bajas y moderadas (Mohanty et al., 2008).

Para la producción de carbón activado se debe partir de una materia prima con propiedades bien definidas tales como abundancia, dureza, estructura inherente de poros, alto contenido de carbono, bajo contenido de ceniza y alto rendimiento en masa durante el proceso de carbonización. Se ha demostrado que materias primas como la madera, el carbón, lignito, endocarpios y huesos de algunas frutas como el coco y las olivas, presentan buenas propiedades para la activación (Ioannidou y Zabaniotou, 2007). Uno de los precursores abundantes en el departamento del Cesar, que reúne las propiedades necesarias para la obtención de carbón activado, es la cáscara o endocarpio de la almendra del fruto de la palma de aceite (Elaeis Guineensis). Existen procesos industriales en donde se generan desechos como el endocarpio de palma y el endocarpio de coco, materiales ricos en carbono con propiedades útiles para la producción de carbón activado, convirtiéndose esto en una buena alternativa para proporcionarle valor agregado a residuos industriales, beneficiando a la agroindustria en el manejo ambiental de desechos (Gómez et al., 2004a, 2004b).

Después de la bonanza algodonera en la década de los 70, la economía del departamento del Cesar ha sido impulsada por la industrialización de la palma africana y el carbón, productos que alcanzaron gran auge alrededor de los años 90. Para el 2005 se contaban con unas 32000 Has de palma africana y se proyecta que para el 2020 el Cesar sea el primer productor nacional con un total de 72000 Has cultivadas. La revista Mundo Cerrejón en su Vol. № 55 del mes de junio de 2008 reporta que, la producción de carbón en Colombia en el 2007 fue de 69.9 millones de ton, siendo el Cesar el mayor productor con un $47.5 \%$ superando a otros departamentos de mayor trayectoria como la Guajira con $43.1 \%$.

Investigaciones desarrolladas a nivel nacional por Ingeominas, la Asociación Colombiana de Ingenieros de minas, la Universidad Nacional de Colombia y Minercol, profundizaron en estudios de algunos mantos de carbones del Cesar, particularmente en muestras de Manto 17 del depósito del municipio de la Jagua de Ibiríco, evidenciando buenos resultados en la obtención de carbón activado. Si bien los resultados encontrados son alentadores para el desarrollo del producto, el estudio no cubrió todos los mantos presentes en la zona por lo que se hace necesario un estudio de otros mantos de los municipios de la Jagua de Ibiríco y la Loma, para estudiar las potencialidades de activación de los carbones con respecto a la activación de algunos desechos de la agroindustria. En este contexto, el trabajo explora la activación de carbones física y químicamente a partir de endocarpio de coco, endocarpio de palma y carbón bituminoso de la cuenca carbonífera del Cesar. Los carbones activados obtenidos se aplican en la adsorción de fenol en fase acuosa, lo cual simula la adsorción de moléculas orgánicas presentes en líquidos de plantas de tratamiento de agua potable y efluentes de procesos industriales. Además, se estudia fundamentalmente la influencia que tienen los grupos superficiales del carbón activado sobre la adsorción de fenol.

\section{METODOLOGÍA}

La metodología experimental aplicada en el desarrollo de la investigación se basó en 4 etapas: i) caracterización de la materia prima; ii) obtención de los carbones activados; iii) caracterización química y textural; y iv) mMétodo experimental de adsorción 


\section{Caracterización de la materia prima}

Se emplearon muestras de carbón mineral, endocarpio de coco y endocarpio de palma, provenientes del Departamento del Cesar, Colombia. Se realizó un análisis próximo al carbón mineral, tomando cantidades entre 0.2 y 0.4 gramos por muestra y empleando el equipo NETZSCH-STA409C (TGA y DTA). Para el análisis de cenizas de los carbones y los endocarpios, se siguió la norma ASTM D 3174, y para los análisis de Humedad residual, Materia volátil y Carbono fijo de los endocarpios, se siguieron las normas ASTM D 3173, 3175 y 3172, respectivamente.

\section{Obtención de los carbones activados}

Las muestras fueron fraccionadas en granos con tamaños comprendidos entre 1.80 y $2.00 \mathrm{~mm}$ y seguidamente se secaron a $110^{\circ} \mathrm{C}$. El tratamiento posterior que se llevó a cabo para cada una de las muestras se describe en la tabla 1. La activación física con $\mathrm{CO}_{2}$ puro, inició con un proceso de carbonización de las muestras en un horno de atmósfera controlada con nitrógeno $\left(\mathrm{N}_{2}\right)$, con el fin de reducir la materia orgánica presente en las mismas (Rodríguez, 2005) para posteriormente activarlas con $\mathrm{CO}_{2}$. En la activación física con vapor de agua, se utilizó como agente activante Nitrógeno humidificado (fracción molar 0.6884 ) con agua a $70^{\circ} \mathrm{C}$ (fracción molar 0.3116). Para la activación química, las muestras de carbón se impregnaron con una solución al 20\% P/V de KOH durante 15 horas a temperatura ambiente y se secaron a $100^{\circ} \mathrm{C}$ durante 3 horas. Las muestras de endocarpio de coco y palma se impregnaron con una solución al $50 \% \mathrm{~V} / \mathrm{V}$ de $\mathrm{H}_{3} \mathrm{PO}_{4}$ bajo las mismas condiciones anteriores; posteriormente, se realizó la carbonización de cada una de las muestras impregnadas bajo un perfil de calentamiento y en atmósfera de nitrógeno. Finalmente las muestras activadas se lavaron con agua destilada caliente hasta obtener un pH entre 6 y 7 , con el objetivo de remover el agente químico impregnado (Rodríguez, 2005). Para los dos tipos de activación el flujo de los gases fue de $150 \mathrm{ml} / \mathrm{min}$, presión de 1 bar y una rampa de calentamiento de $10^{\circ} \mathrm{C} / \mathrm{min}$.

En la tabla 1, se muestran los tratamientos de activación de los precursores lignocelulósicos, endocarpio de coco, endocarpio de palma africana, y de los mantos 45, 200 y 240 . Las muestras se identificaron teniendo en cuenta que las primeras letras indican el material activado: Manto (M), seguido de su respectivo número $(45,200$ y 240) y, los precursores lignocelulósicos: Endocarpio o cáscara de coco (CC) y Endocarpio o cuesco de palma (CP); las ultimas letras indican el tipo de activación física (F) y química (Q), y el agente activante: dióxido de carbono (D) y vapor (V).

Tabla 1: Denominación de los materiales activados

\begin{tabular}{lcccc}
\hline $\begin{array}{l}\text { Material } \\
\text { precursor }\end{array}$ & $\begin{array}{c}\text { Tratamiento de } \\
\text { activación }\end{array}$ & $\begin{array}{c}\text { Condiciones de } \\
\text { Carbonización }\left({ }^{\circ} \mathrm{C} / \mathrm{h}\right)\end{array}$ & $\begin{array}{c}\text { Condiciones de } \\
\text { Activación }\left({ }^{\circ} \mathrm{C} / \mathrm{h}\right)\end{array}$ & Denominación \\
\hline Manto 45 & $\mathrm{CO}_{2}$ & $800 / 2$ & $800 / 4$ & $\mathrm{M} 45-\mathrm{FD}$ \\
\hline Manto 200 & $\mathrm{CO}_{2}$ & $800 / 2$ & $800 / 4$ & $\mathrm{M} 200-\mathrm{FD}$ \\
\hline Manto 240 & Vapor de Agua & $800 / 2$ & $800 / 3$ & $\mathrm{M} 240-\mathrm{FV}$ \\
& $\mathrm{CO}_{2}$ & $800 / 2$ & $800 / 4$ & $\mathrm{M} 240-\mathrm{FD}$ \\
& $\mathrm{KOH}$ & --- & $900 / 1$ & $\mathrm{M} 240-\mathrm{QB}$ \\
\hline Endocarpio de & Vapor de Agua & $600 / 2.5$ & $800 / 3$ & $\mathrm{CC}-\mathrm{FV}$ \\
Coco & $\mathrm{CO}_{2}$ & $600 / 2.5$ & $900 / 1.5$ & $\mathrm{CC}-\mathrm{FD}$ \\
& $\mathrm{H}_{3} \mathrm{PO}_{4}$ & --- & $500 / 2$ & $\mathrm{CC}-\mathrm{QA}$ \\
\hline Endocarpio de & $\mathrm{Vapor} \mathrm{de} \mathrm{Agua}$ & $600 / 2.5$ & $800 / 3$ & $\mathrm{CP}-\mathrm{FV}$ \\
Palma Africana & $\mathrm{CO}_{2}$ & $600 / 2.5$ & $900 / 1.5$ & $\mathrm{CP}-\mathrm{FD}$ \\
& $\mathrm{H}_{3} \mathrm{PO}_{4}$ & --- & $500 / 2$ & $\mathrm{CP}-\mathrm{QA}$ \\
\hline
\end{tabular}

En la figura 1, se muestra un esquema del horno de atmósfera controlada para la obtención de los carbones activados, que funciona con un controlador de temperatura Watlow ${ }^{\circledR}$. Igualmente, el horno permite regular el tipo, presión y flujo de gases de carbonización y activación, mediante un tablero de control de gases; además, el diseño del equipo permite intercambiar el reactor metálico (activación química), por el reactor de vidrio de cuarzo (activación física). 


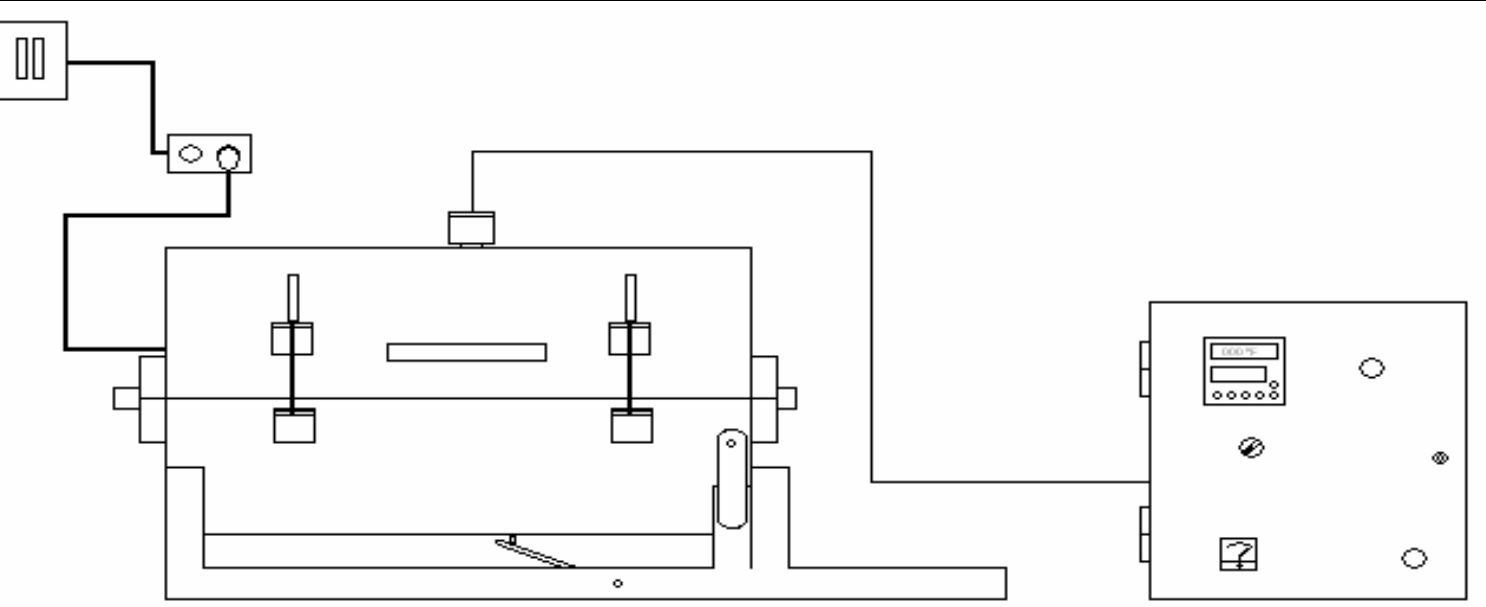

Temperatura máxima: $1200^{\circ} \mathrm{C}$

Cámara útil: $12 \times 40 \mathrm{~cm}^{2}$

Potencia máxima: $1400 \mathrm{~W}$

Tensión: 200V

Numero de resistencia: dos con seis tramos c/u

Resistencia tipo Kanthal A-1(54 espiras por

tramo de $8 \mathrm{~mm}$ )

Tablero de control digital configurable Watlow
Manómetro de U-30 psi

Un rotámetro para Nitrógeno y uno para $\mathrm{CO}_{2}$ con sus respectivas válvulas de regulación de flujo incorporadas

Bomba peristáltica:

Flujo máximo: $2900 \mathrm{ml} / \mathrm{min}$

Flujo mínimo: $1.7 \mathrm{ml} / \mathrm{min}$

Fig. 1: Horno de activación

\section{Caracterización química y textural}

Los carbones activados obtenidos fueron caracterizados determinando el área superficial BET, el volumen y distribución de poros. El equipo utilizado para la obtención de las isotermas de $\mathrm{N}_{2}$ fue el sortómetro Quantachrome Nova 3200 y se usó el programa Autosorb para establecer las correlaciones correspondientes. El área BET (SBET) reporta el área total aportada por los micro y mesoporos a partir de los datos obtenidos de la rama de adsorción de la isoterma de $\mathrm{N}_{2}$ a $77 \mathrm{~K}$ (Zapata et al., 2005). Para la determinación de los grupos superficiales del carbón activado se utilizó la titulación ácido-base propuesta por Boehm (Boehm, 1966).

\section{Método experimental de adsorción}

Para establecer la longitud de onda de máxima absorción en la determinación de fenol en solución acuosa, se realizó un barrido entre 200 y $400 \mathrm{~nm}$ a una solución de $20 \mathrm{mg} / \mathrm{L}$ de fenol con pH 6, usando un espectrofotómetro UV-VIS Thermospectronic Genesys 5.

El tiempo de equilibrio se determinó con soluciones de mínima y máxima concentración, 30 y 500 $\mathrm{mg} / \mathrm{L}$ respectivamente, de cada uno de los solutos a un $\mathrm{pH}$ de 7.0. Se colocaron $50,0 \mathrm{~mL}$ de cada una de las soluciones en recipientes cerrados, a los cuales se les agregó 0,50g de carbón activado; a dichas soluciones se les determinó la medida de absorción de fenoles a través del tiempo desde 0 a 48 horas.

Se colocaron $0,50 \mathrm{~g}$ del material carbonoso y 50,0 mL de la solución fenólica de concentración conocida, dentro de un erlenmeyer cerrado a pH 6 con agitación constante y temperatura de $25,0{ }^{\circ} \mathrm{C}$ de 0 a 48h. Al mismo tiempo se realizó un control y ajuste de la medida de $\mathrm{pH}$ empleando soluciones de hidróxido de sodio y ácido clorhídrico 0,01M. Al finalizar el periodo establecido se midió el pH y la absorbancia de la solución. 


\section{RESULTADOS Y DISCUSIÓN}

En la tabla 2 se reportan los resultados del análisis próximo para cada una de las muestras.

Tabla 2: Análisis próximo de las materias primas en base seca (Bs) y base húmeda (Bh)

\begin{tabular}{lccccccc}
\hline $\begin{array}{l}\text { Materia } \\
\text { Prima }\end{array}$ & $\begin{array}{c}\text { Ceniza } \\
(\mathrm{Bh}) \\
(\%)\end{array}$ & $\begin{array}{c}\text { Ceniza } \\
(\mathrm{Bs})\end{array}$ & $\begin{array}{c}\text { Humedad } \\
(\%)\end{array}$ & $\begin{array}{c}\text { Materia } \\
\text { Volátil }(\mathrm{Bh}) \\
(\%)\end{array}$ & $\begin{array}{c}\text { Materia Volátil } \\
(\mathrm{Bs})(\%)\end{array}$ & $\begin{array}{c}\text { Carbono } \\
\text { Fijo (Bh) } \\
(\%)\end{array}$ & $\begin{array}{c}\text { Carbono } \\
\text { Fijo (Bs) } \\
(\%)\end{array}$ \\
\hline M45 & 13.08 & 13.71 & 3.75 & 30.61 & 32.08 & 51.73 & 54.21 \\
M200 & 9.91 & 10.79 & 7.51 & 28.98 & 31.57 & 52.92 & 57.64 \\
M240 & 4.02 & 4.35 & 6.96 & 27.69 & 29.95 & 60.74 & 65.70 \\
CC & 1.30 & --- & 8.50 & 68.70 & --- & 21.50 & --- \\
CP & 1.60 & --- & 7.30 & 72.70 & --- & 18.40 & -- \\
\hline
\end{tabular}

Los resultados del análisis próximo de los carbones minerales ubican a los carbones usados en este estudio dentro del rango de los Carbones Bituminosos (UPME, 2005). Teniendo en cuenta estos resultados se observa que, por su contenido de carbono fijo, los materiales son aptos o reúnen las condiciones necesarias para ser activados.

En la Tabla 3 se muestran los resultados de los valores de las áreas superficiales BET y el Volumen de poro determinados para cada una de las muestras activadas físicamente (Dióxido de carbono y Vapor de Agua) y químicamente $\left(\mathrm{KOH}\right.$ y $\left.\mathrm{H}_{3} \mathrm{PO}_{4}\right)$.

Tabla 3: Valores de Área superficial BET y Volumen de poro de las muestras

\begin{tabular}{lccc}
\hline Muestra & Peso $(\mathrm{g})$ & $\begin{array}{c}\text { Área } \\
\text { superficial } \\
\left(\mathrm{m}^{2} / \mathrm{g}\right)\end{array}$ & $\begin{array}{c}\text { Volumen poro } \\
(\mathrm{cc} / \mathrm{g})\end{array}$ \\
\hline M45-FD & 0.139 & 43.69 & 0.0228 \\
M200-FD & 0.186 & 179.25 & 0.0864 \\
M240-FD & 0.125 & 177.79 & 0.0852 \\
M240-FV & 0.186 & 217.79 & 0.1018 \\
CC-FV & 0.159 & 611.77 & 0.2392 \\
CC-FD & 0.114 & 1208.94 & 0.5186 \\
CP-FV & 0.135 & 543.12 & 0.2373 \\
CP-FD & 0.150 & 737.28 & 0.3076 \\
\hline M240-QB & 0.122 & 56.94 & 0.0279 \\
CC-QA & 0.143 & 701.98 & 0.2875 \\
CP-QA & 0.147 & 391.71 & 0.1631 \\
\hline
\end{tabular}

Para las muestras activadas físicamente la que reportó mayor área superficial fue la CC-FD con $1208.94 \mathrm{~m}^{2} / \mathrm{g}$ y un volumen de poro de $0.5186 \mathrm{cc} / \mathrm{g}$, para las muestras activadas químicamente, se obtuvo un área de $701.98 \mathrm{~m}^{2} / \mathrm{g}$ y volumen de poro de $0.2875 \mathrm{cc} / \mathrm{g}$ para la muestra CC-QA.

En las Figuras 2 y 3 se grafican las Isotermas con nitrógeno y la distribución de poros de algunas de las muestras activadas obtenidas, respectivamente.

En las isotermas de adsorción con $\mathrm{N}_{2}$ a $77 \mathrm{~K}$ presentadas en la Figura 2, se observa que todos los carbones forman el codo de las isotermas de adsorción tipo 1 o Langmuir a presiones relativas 
cercanas a 0.1 , este tipo de isoterma es característica de sólidos microporosos, muy comunes en carbones activados, en donde el llenado de los poros se da en una sola capa o monocapa; para este tipo de isoterma, cada poro del sólido puede albergar una sola molécula (molécula de $\mathrm{N}_{2}$ ), teniendo los poros igual actividad para la adsorción (Bandosz, 2006).

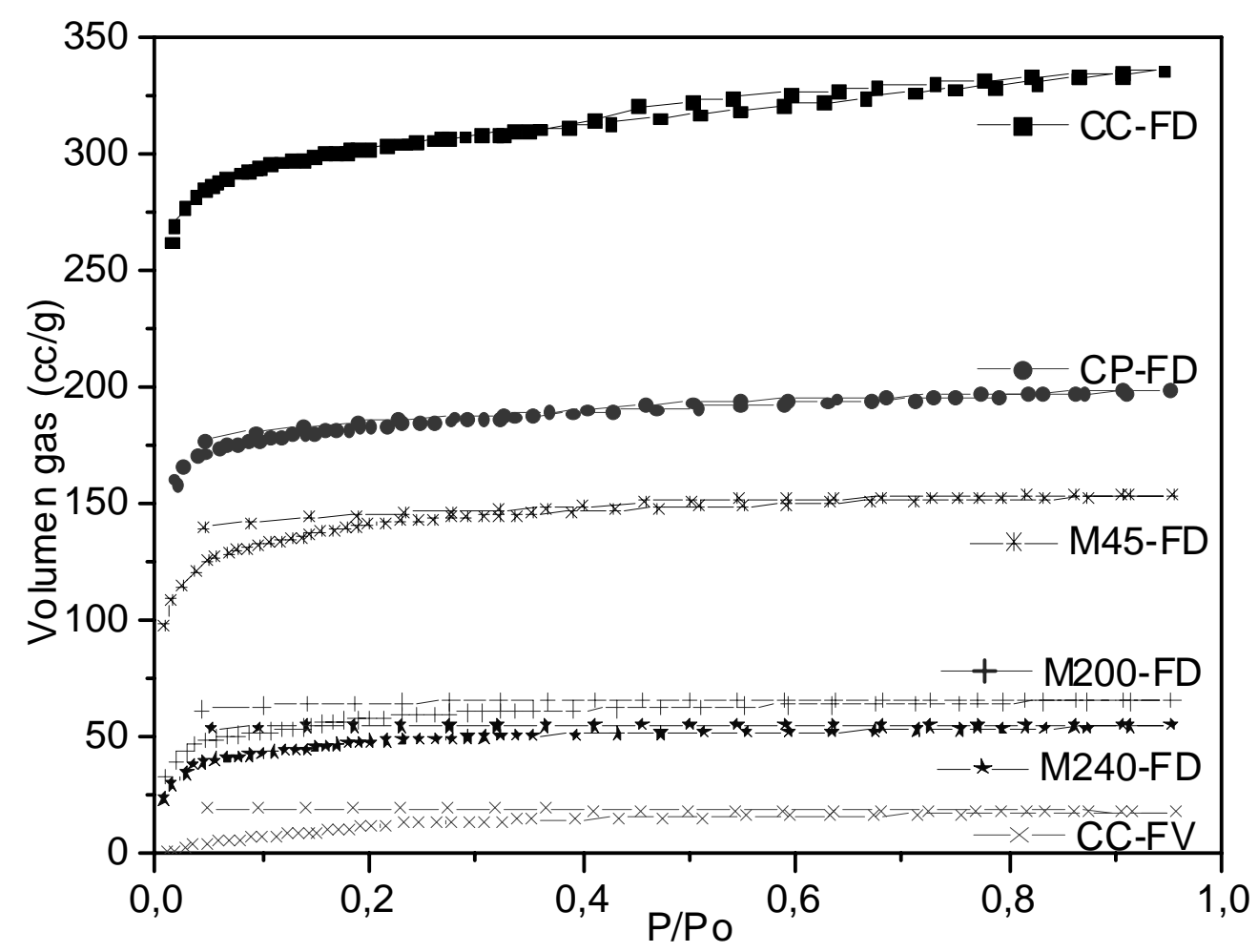

Fig. 2: Isotermas de adsorción con $\mathrm{N}_{2}$ a $77 \mathrm{~K}$ de los carbones activados

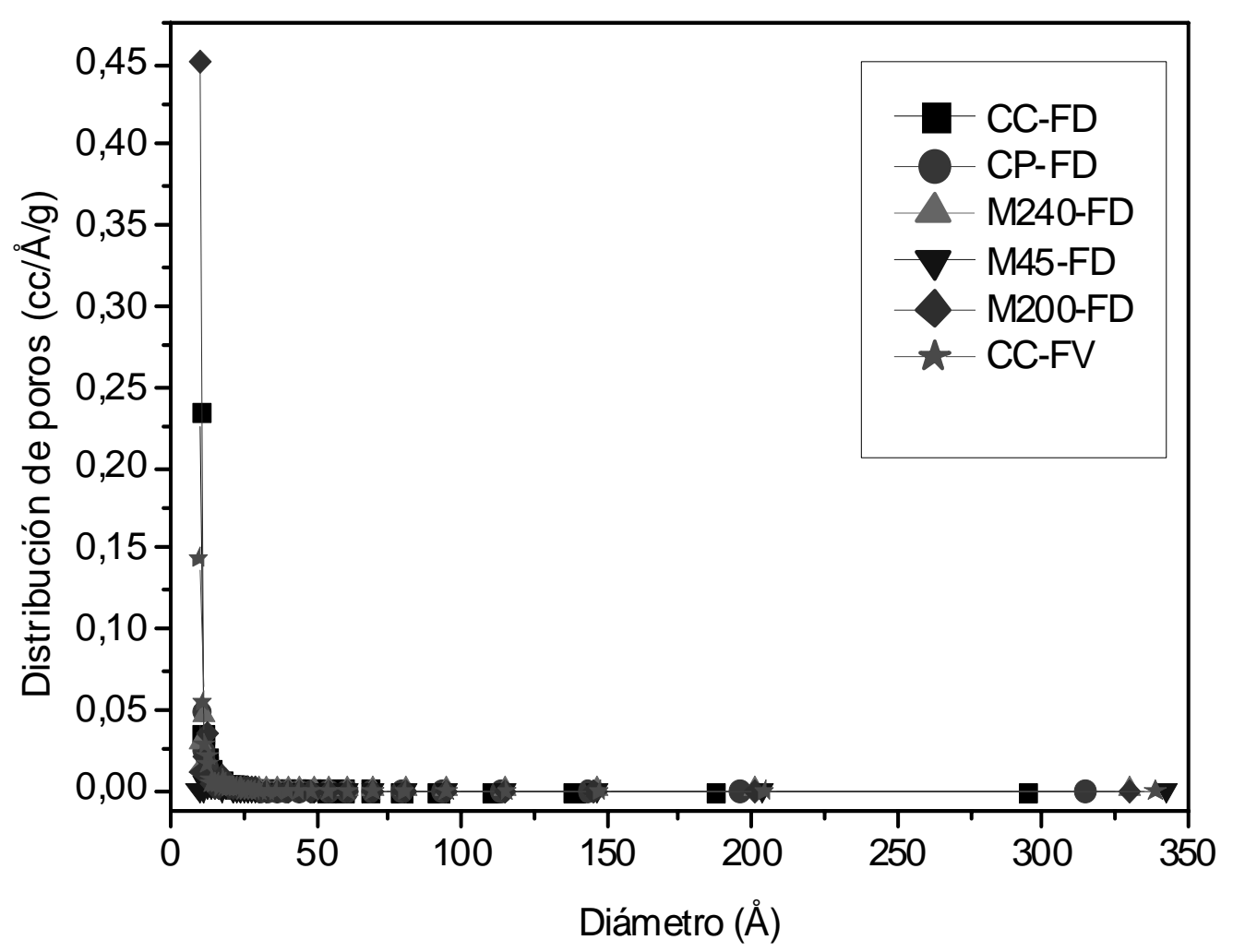

Fig. 3: Distribución de Poros Método BJH 
En la Figura 3 se observa que los carbones activados obtenidos son de carácter microporoso debido a que la adsorción del gas (Nitrógeno) ocurrió en los poros inferiores a los $20 \AA$ (<2nm). En la Tabla 4 se presentan los resultados obtenidos mediante el método de titulación propuesto por Boehm para algunas de las muestras activadas, con el cual se cuantifica el contenido de grupos de carácter ácido (Carboxilos, Lactonas y Fenoles) y básico.

Tabla 4: Resultados del Método de Boehm

\begin{tabular}{l|c|c}
\hline \multirow{2}{*}{ CARBONES } & \multicolumn{2}{|c}{ GRUPOS QUÍMICOS SUPERFICIALES } \\
\cline { 2 - 3 } & $\begin{array}{c}\text { GRUPOS ÁCIDOS } \\
\left(\mathrm{meqg}^{-1}\right)\end{array}$ & $\begin{array}{c}\text { GRUPOS BÁSICOS } \\
\left(\mathrm{meqg}^{-1}\right)\end{array}$ \\
\hline M240-FV & 0.105 & 0.034 \\
\hline M240-FD & 0.010 & 0.041 \\
\hline M240-QB & 0.054 & 0.142 \\
\hline CP-FV & 0.008 & 0.206 \\
\hline CP-FD & 0.055 & 0.161 \\
\hline CC-FD & 0.043 & 0.138 \\
\hline
\end{tabular}

Los resultados obtenidos para el contenido de grupos ácidos totales de las muestras de carbones activados presentan valores de acidez en un rango aproximado de 0.07 a $0.105 \mathrm{meqg}^{-1}$, rango que permite observar las diferencias entre éstos; la basicidad total varía entre 0.041 y 0.206 meqg $^{-1}$ que muestra el carácter básico de los carbones activados obtenidos térmicamente en las condiciones descritas.

Para el procedimiento de adsorción de fenol sobre los carbones activados los resultados son presentados en la tabla 5, en donde los valores de Co, Ce y Qe representan la concentración inicial de la solución de fenol, concentración en el equilibrio del contaminante y cantidad adsorbida del contaminante, respectivamente.

Tabla 5: Capacidad de adsorción de las muestras

\begin{tabular}{lccrr}
\hline Muestra & Peso Muestra (g) & Co $(\mathrm{ppm})$ & $\begin{array}{r}\mathrm{Ce}^{*} \\
(\mathrm{ppm})\end{array}$ & \multicolumn{1}{c}{$\begin{array}{c}\mathrm{Qe}^{* *} \\
(\mathrm{mg} / \mathrm{g})\end{array}$} \\
\hline CP-FV & 0.206 & 500 & 287.07 & 20.67 \\
CP-FD & 0.205 & 500 & 21.36 & 46.58 \\
CC-FD & 0.204 & 500 & 6.67 & 48.41 \\
M240-FV & 0.208 & 500 & 463.95 & 3.47 \\
M240-FD & 0.206 & 500 & 293.33 & 20.08 \\
M240-QB & 0.205 & 500 & 12.18 & 47.47 \\
\hline
\end{tabular}

De los resultados presentados en la Tabla 5, se puede observar la alta capacidad de adsorción de fenol de los carbones activados obtenidos por activación física con $\mathrm{CO}_{2}$ para endocarpio de coco y endocarpio de palma, como también el obtenido por activación química con $\mathrm{KOH}$ del manto 240, esto significa que estos materiales adsorbentes tienen alta probabilidad de ser empleados en tratamientos de aguas potables o residuales y brindar un buen desempeño en los sistemas de adsorción utilizados. La mayor capacidad de adsorción de fenol en solución acuosa se debe a la cantidad de fenol que se adsorbe en los microporos, en las partículas de menor diámetro se tiene una mayor disponibilidad del área de los microporos y por esta razón, la adsorción del fenol en solución acuosa aumenta (Marsh y Rodríguez, 2006). 
Los resultados de la capacidad de adsorción de fenol $(48 \mathrm{mg} / \mathrm{g}$ ) presentan un comportamiento similar al obtenido en otros trabajos realizados a nivel nacional (Moreno et al., 2007a, 2007b) cuyo rango de capacidad de adsorción de fenol se encuentra en el mismo orden.

La figura 4 presenta la relación entre la cantidad adsorbida de fenol en los carbones activados en función del área superficial de éstos; en los resultados se observa que los carbones que presentan la mayor adsorción de fenol son los que tienen menor y mayor área superficial, es decir, M240-QB y CC-FD, respectivamente. La mayor adsorción se obtuvo para el carbón activado CC-FD que tiene como precursor endocarpio de coco y que presenta la mayor área superficial $\left(1209 \mathrm{~m}^{2} \mathrm{~g}^{-1}\right)$ del conjunto de sólidos empleados. Sin embargo, a nivel general los resultados indican que el área superficial del material adsorbente no lleva una relación directa con la adsorción del fenol en los carbones activados.

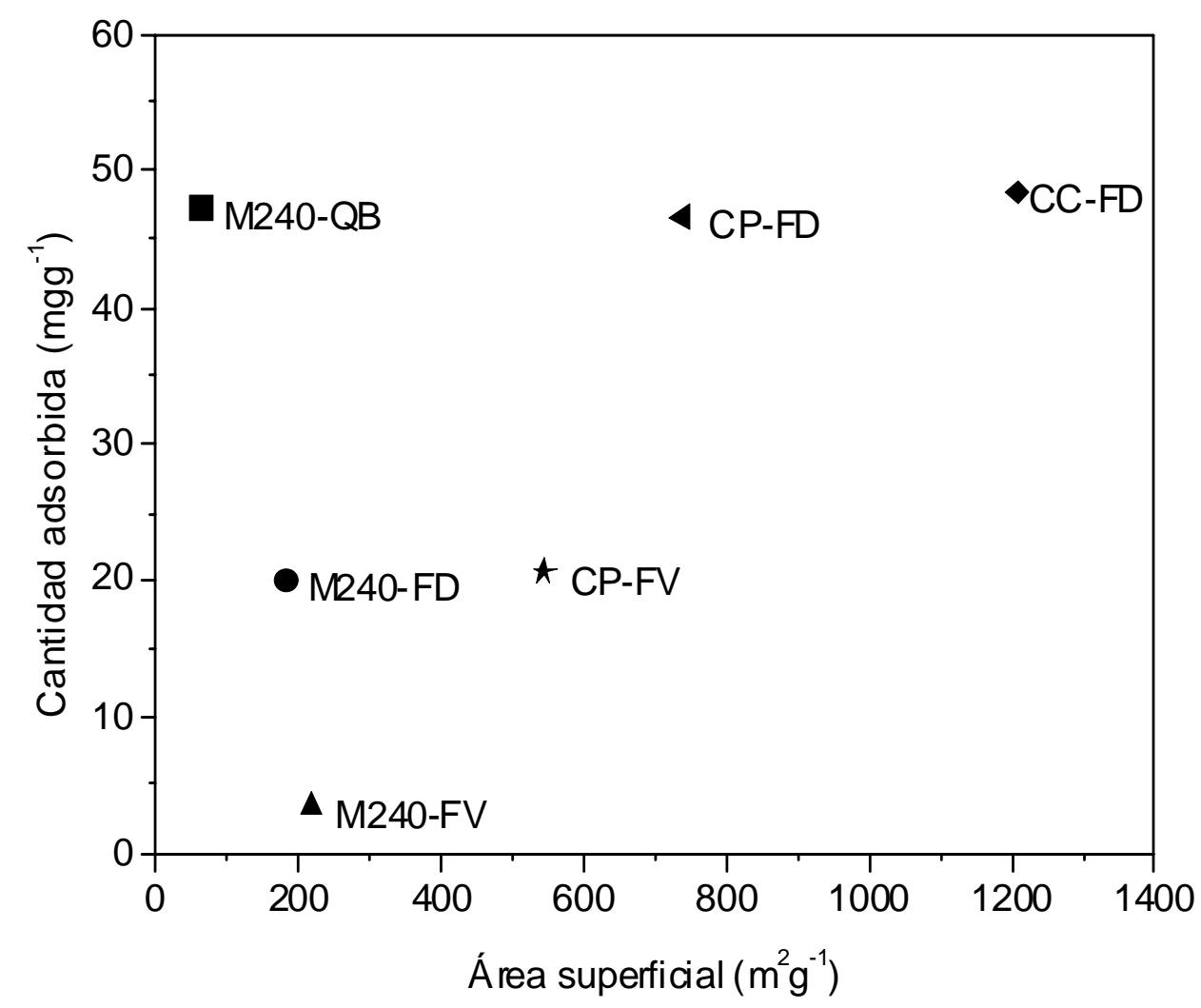

Fig. 4: Cantidad adsorbida de Fenol en función del área superficial de los carbones activados

Las propiedades adsorbentes de un carbón activado no dependen únicamente de la superficie y la porosidad. La presencia de oxígeno, hidrógeno y nitrógeno en forma de grupos funcionales en la superficie del carbón ejerce un gran efecto en las propiedades adsorbentes, especialmente frente a moléculas polares o polarizables (Rodríguez, 2007).

Se conoce que en el caso de la retención de fenoles en agua, un aumento del grado de oxidación del carbón conduce a un descenso de la superficie del carbón ocupada por el fenol (Rodríguez, 2007). Algunos autores han comprobado que la presencia de estructuras tipo ácido carboxílico y lactonas son las principales responsables del descenso, y se debe a la preferencia de las moléculas de agua respecto al soluto por estos grupos hidrofílicos, ocupándose así una parte importante de la superficie del carbón (Moreno, 2004). Esto indica que la adsorción de aniones sobre carbón activado ocurre principalmente en los sitios básicos del carbón activado (Leyva, 2007). Por tanto, los resultados obtenidos confirman lo anterior, debido a que los carbones activados desarrollados en este trabajo son de carácter básico, favoreciendo la adsorción de fenol en solución acuosa. 


\section{CONCLUSIONES}

Los carbones bituminosos del Departamento del Cesar al ser activados desarrollaron áreas superficiales entre 44 y $218 \mathrm{~m}^{2} / \mathrm{g}$, el manto 240 activado química y físicamente, reportó valores de 57 y $218 \mathrm{~m}^{2} / \mathrm{g}$, respectivamente. Con todo esto, el manto 240 sometido a activación química presentó el mejor resultado en la prueba de adsorción de fenol, evidenciando que el área superficial del material adsorbente no es el factor determinante en la adsorción de fenol sobre los carbones activados.

El endocarpio del coco es un material que reúne las propiedades necesarias para producir carbones activados debido a que desarrolló un área superficial muy interesante que sobrepasa los $1000 \mathrm{~m}^{2} / \mathrm{g}$, siendo altamente competitivo con los carbones activados comerciales producidos a partir de biomasa.

La química superficial de los carbones activados obtenidos favorece la cantidad de compuestos fenólicos adsorbidos. Igualmente, el buen comportamiento que presentaron los carbones en la adsorción del fenol en solución acuosa se debe al carácter microporoso de los mismos.

\section{AGRADECIMIENTOS}

A COLCIENCIAS por su apoyo financiero, al CIDI en la Universidad Popular del Cesar por su apoyo de infraestructura, al Doctor Juan Carlos Moreno de la Universidad de los Andes, al Ingeniero Ángel Polo y demás investigadores del proyecto, por sus aportes académicos y sugerencias.

\section{REFERENCIAS}

Bandosz, T.; Activated Carbon Surfaces in Environmental Remediation. Interface science and technology, Elsevier, New York: 7(3), 107-153 (2006).

Boehm, H.P.; Advances in Catalysis. Eley, D.D.H. Pines., P.B. Weisz, Segunda Edición, p. 192, Academic Press, New York, USA (1966).

Gómez, A., W. Klose, S. Rincón y W. Wiest; Proceso de producción de carbón activado de cáscaras de palma de aceite en un horno rotatorio y su aplicación en la limpieza de NO. Palmas: 25(2), 461471 (2004a).

Gómez, A., W. Klose, S. Rincón y W. Wiest; Transformación termoquímica de la biomasa residual del proceso de extracción del aceite de palma: tecnologías y perspectivas. Palmas: 25(2), 388-397 (2004b).

Ioannidou, O. y A. Zabaniotou; Agricultural residues as precursors for activated carbon production - A review. Renewable and Sustainable Energy Reviews: 11(9), 1966-2005 (2007).

Leyva, R.; Importancia y Aplicaciones de la Adsorción en Fase Líquida, En Sólidos Porosos. Preparación, Caracterización y Aplicaciones por J. C. Moreno-Piraján, p. 155-207, Ediciones Uniandes, Bogotá, Colombia (2007).

Marsh, H. y F. Rodríguez; Activated Carbon. Elsevier, España, p. 536 (2006).

Mohanty, K., D. Das y M.N. Biswas; Utilization of Arachis hypogaea hull, an agricultural waste for the production of activated carbons to remove phenol from aqueous solutions. Journal of Environmental Science and Health, Part B: Pesticides, Food Contaminants, and Agricultural Wastes, 1532-4109: 43(5), 452-463 (2008).

Moreno, C.; Eliminación de contaminantes orgánicos de las aguas mediante adsorción en materiales de carbón. En: Rodríguez Reinoso, F. Adsorbentes en la solución de algunos problemas ambientales, Madrid: Cyted, Cap. 4 p. 53-77 (2004). 
Moreno, J., L. Navarrete, L. Giraldo y V. Garcia; Adsorción de Fenol y 3-Cloro Fenol sobre Carbones Activados mediante Calorimetría de Inmersión. Inf. tecnol. [online]: 18(3), 71-80 (2007a).

Moreno, J., V. Sarria, A. Polo y L. Giraldo; Evaluación del Peróxido de Hidrógeno en la Oxidación de Fenol con Hierro Soportado Sobre Tela de Carbón Activado. Inf. tecnol. [online]: 18(2), 67-72 (2007b).

Rodríguez, F. Carbón activado: estructura, preparación y aplicaciones, Revista Uniandes p. 66-69 (2005).

Rodríguez, F.; El Carbón activado como adsorbente universal. En Sólidos Porosos. Preparación, Caracterización y Aplicaciones por J. C. Moreno-Piraján, Ediciones Uniandes, Bogotá, Colombia. p. 1-42 (2007).

UPME. Unidad de Planeación Minero Energética. La cadena del Carbón. El carbón Colombiano: Fuente de Energía para el Mundo. Ministerio de Minas y Energía, Bogotá, Colombia. p. 7-52 (2005).

Zapata, Z. y otros cinco autores; Producción de carbón activado a partir de carbón subbituminoso en reactores de lecho fluidizado por proceso autotérmico. Dyna: 72(147), 47-56 (2005). 